\title{
Controlling of jerk chaotic system via linear feedback control strategies
}

\author{
Karam Adel Abed \\ Department operations Research and intelligence techniques, College of Computer Sciences and Mathematics, \\ University of Mosul, Iraq
}

\begin{tabular}{lll} 
Article Info & ABSTRACT \\
Article history: & $\begin{array}{l}\text { In this paper, the strategies of linear feedback control for jerk system is } \\
\text { considered. These strategies consist of four strategies (ordinary feedback } \\
\text { Received Dec 24, } 2019\end{array}$ & $\begin{array}{l}\text { control, dislocated feedback control, speed feedback control, and enhancing } \\
\text { feedback control). We propose to combine between these strategies and } \\
\text { Revised Mar 26, 2020 } \\
\text { obtained a better result from this combine. Numerical solution achieved the } \\
\text { same results. }\end{array}$ \\
\hline
\end{tabular}

Keywords:

Dislocated

Enhancing feedback control

Jerk system

Ordinary

Speed

Strategies of linear feedback

Copyright $(0) 2020$ Institute of Advanced Engineering and Science. control

All rights reserved.

Corresponding Author:

Karam Adel Abed,

Department operations Research and intelligence techniques,

College of Computer Sciences and Mathematics,

University of Mosul, Mosul, Iraq.

Email:karamadel@uomosul.edu.iq

\section{INTRODUCTION}

More recently, scientists have focused on controlling chaos because of their practical and engineering applications to physics, chemical reactor, control theory, biological networks, artificial neural networks, telecommunications and secure communication [1-4]. Control of disorder is one of the most important topics in nonlinear dynamic science [5,6]. The first to introduce codification in the control of the disorder was in 1990 [7] In order to succeed in controlling chaos many different methods and techniques have been submit such as : adaptive control method, running control method, differential geometric method, sliding method control, $\infty \mathrm{H}$ control, and so on [8] Generally there are two prime methods for controlling chaos: the first feedback control and the second non-feedback control. The feedback control way bid many properties like robustness and computational complexity over the non-feedback control method [9-13]. In 1963 Lorenz accidentally discovered the chaotic behaviour of the regime when he was designing a threedimensional model of weather forecasting [14-17]. In 1976 Rossler built many chaotic three-dimensional autonomous systems, and Gebria was easier than the Lorenz system [18,19]. In 2016 (Zhang and Han) succeeded in building an independent chaotic system with cubic nonlinearity [20-22]. In the same year, both Vaidyanathan and Volos managed to build a novel conservative jerk chaotic system with two cubic nonlinearities and debate its adaptive backstepping control [23, 24].

\section{PROBLEMFORMULATION OUR METHODOLOGY}

If we have a class of nonlinear dynamical system has the form [25-27]: 


$$
\dot{V}=A V+F(V)
$$

Where $V(t)=\left[v_{1}, v_{2}, \ldots \ldots \ldots, v_{n}\right]^{T} \in R^{n * 1}, i=1,2,3, \ldots, n$, is the state variables of the system A= (aij)n*n is the matrix of their system parameters. The control system is described by the next formula:

$$
\dot{V}=A V+Y
$$

Where $Y=\left[y_{i}\right]^{T}=\left[y_{1}, y_{2}, \ldots \ldots, y_{n}\right]^{T} \in R^{n * 1}$ represent the control, the purpose of the control problem is to select suitable a feedback controller $(\mathrm{Y})$ to make that $\lim _{t \rightarrow \infty}\|V(t)\|=0$. This control $(\mathrm{Y})$ based on linear feedback control strategies which consist of four sub strategies: ordinary feedback control, dislocated feedback control, speed feedback control and enhancing feedback control, and can be written in a succinct mathematical formula as [25]:

$$
Y=y_{i}=\left\{\begin{array}{c}
-m v_{i}: \text { if } i=j(\text { ordinary }) \\
-m v_{j}: \text { if } i \neq j(\text { dislocated }) \\
-m v_{i}: \text { if } i \neq j(\text { speed }) \\
-m\left[v_{i}\right]_{i=1}^{n}: \text { if } i=j(\text { enhancing })
\end{array}\right.
$$

Where $(\mathrm{m})$ is called a feedback coefficient, and $\mathrm{m}>0$. In some times, we produce at two least positive feedback coefficient according to the Routh Hurwitz criterion. Therefore, we will use the following formula to determine the active feedback coefficient [12]:

$$
m=\bigcap_{i=1}^{n} m_{i}=m_{1} \cap m_{2} \cap \ldots \ldots \cap m_{n}
$$

Depending on the above sub-strategies, the nonlinear system can be control if it has positive feedback coefficient. This is a sufficient and necessary condition for controlled system dynamic nonlinear.

\section{DESCRIPTION SYSTEM}

In this section a new dynamical system which called jerk chaotic is consider. This system contains 7 borders of which two of nonlinear. the chaotic system can be described by the next mathematical model:

$$
\left\{\begin{array}{c}
\dot{x}=\mathrm{y} \\
\dot{y}=\mathrm{z} \\
\dot{\mathrm{z}}=-\alpha \mathrm{z}-\beta \mathrm{x}+\delta \mathrm{y}+\mathrm{xy}^{2}-\mathrm{x}^{3}
\end{array}\right.
$$

where $\alpha, \beta, \delta$ are positive constant, and it has only one equilibrium point $O(0,0,0)$, the system chaotic attractor when the parameter $\alpha=3.6, \beta=1.3, \delta=0.1$, the Figure 1 and 2 shows the attractor of the system (5) in (2-D) and (3-D) respectively.

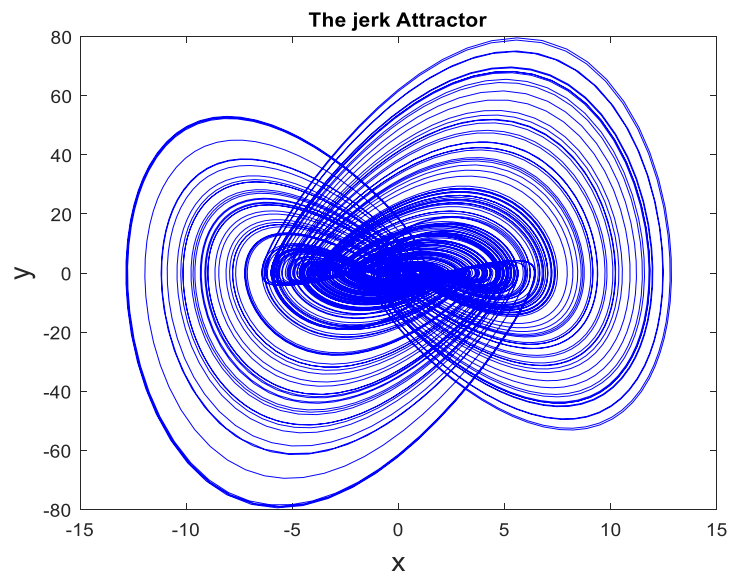

Figure 1. The attractor of the system (5) in 2dimensional

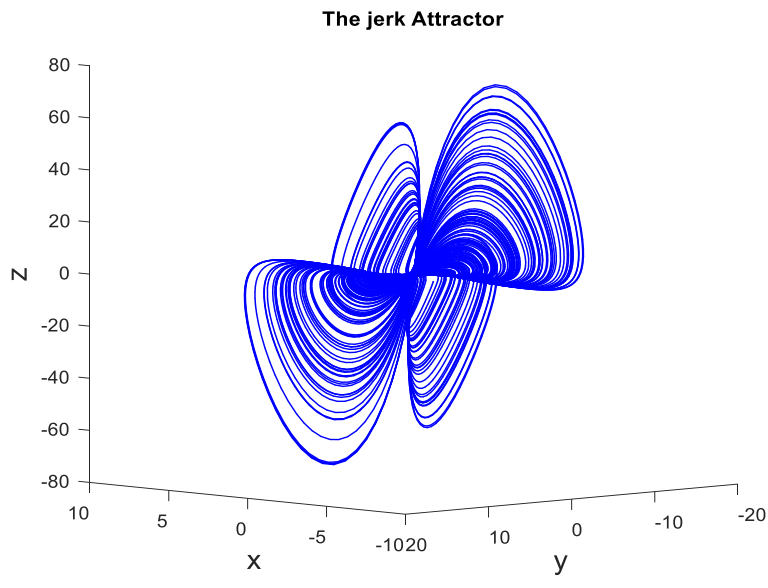

Figure 2. The attractor of the system (5) in 3dimensional 


\section{HELPING RESULTS}

The control problem with a positive feedback coefficient for nonlinear dynamical system based on strategies of linear feedback control (ordinary, dislocated, speed, enhancing), is summarise by the following theory.

Theorem: Control matter with a positive feedback coefficient have the next cases [25]:

1. control it if there is only one positive feedback gian $\mathrm{m}$, and $(\mathrm{m}>$ constant).

2. not control if there is only one positive feedback gian $\mathrm{m}$, and $(\mathrm{m}<$ constant $)$.

3. control it, if there is at two least positive feedback coefficient and $\cap_{i=1}^{n} m_{i} \neq \varnothing$.

4. not control, if there is at two least positive feedback coefficient and $\cap_{i=1}^{n} m_{i}=\emptyset$.

\subsection{Ordinary feedback control}

When the Jerk system is resolved within this strategy, we get three strategies, only one strategy succeeds.

Theorem: when add the control, $U_{2}=-m x_{2}$, then the active a positive feedback coefficient is $m>0.1247$

Proof: From a new control with system (5) we obtain the next form:

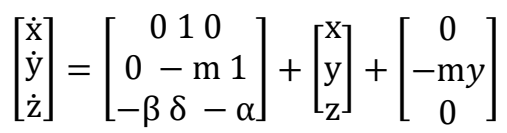

And the auxiliary equation of above system is:

$$
\tau^{3}+\underbrace{(\alpha+m)}_{a} \tau^{2}+\underbrace{(\alpha m-\delta)}_{b} \tau+\underbrace{\beta}_{c}=0
$$

Now based on Routh-Hurwitz theorem the first and the condition is satisfied:

$$
\begin{aligned}
& a>0 \Rightarrow(\alpha+m)>0 \\
& c>0 \Rightarrow \beta>0
\end{aligned}
$$

Finally, from the third condition $a b-c>0$, we get the following inequality:

$\alpha m^{2}+\left(\alpha^{2}-\delta\right) m-\alpha \delta-\beta>0$

Now, solving this inequality two feedback coefficients are obtained:

$$
m_{1}>0.1247, m_{2}>-3.6969
$$

the active is $m_{1}>0.1247$.

Therefore, then satisfied the first condition of theorem. the prove completed. the following analytical and simulation numerical are justified the same result.

Analytically: by substitute the parameter of value $\alpha=3.6, \beta=1.3, \delta=0.1$ in the equation (6) and chosen positive feedback coefficient as the following:

$$
\begin{aligned}
& m=0.123 \text { then, } \tau_{1,2}=0.00083 \mp 0.5907 i, \tau_{3}=-3.7247 \\
& m=0.1247 \text { then }, \tau_{1,2}=0, \tau_{3}=-3.7247 \\
& m=0.125 \text { then }, \tau_{1,2}=-0.000131 \pm 0.5907 i, \tau_{3}=-3.7247
\end{aligned}
$$

\section{Numerically:}

Depending on the MATLAB (17.a) program and the choice of parameter values $(\alpha=3.6, \beta=$ $1.3, \delta=0.1)$ and the use of Rang Kuta of the fourth rank and the step $(\Delta t=0.1)$ and the initial $\left(\mathrm{X}_{\mathrm{o}}, \mathrm{Y}_{\mathrm{o}}, \mathrm{Z}_{\mathrm{o}}\right)$ values $(1,0,-1)$, to check the control system (5) with controller $\left(u_{2}=-m y\right)$, the linear feedback coefficient was selected $(m=0.123$ and $m=0.125)$ respectively. Figure 3 illustrates the behavior of variables $(\mathrm{x}, \mathrm{y}, \mathrm{z})$ and we observe the spacing in Figure 3(a), we observe the convergence in Figure 3(b), as shown in the next Figure 3. 
(a) $m=0.123$

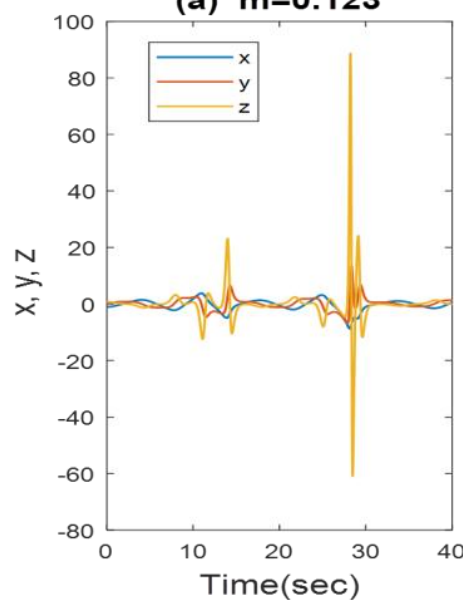

(b) $\mathrm{m}=0.126$

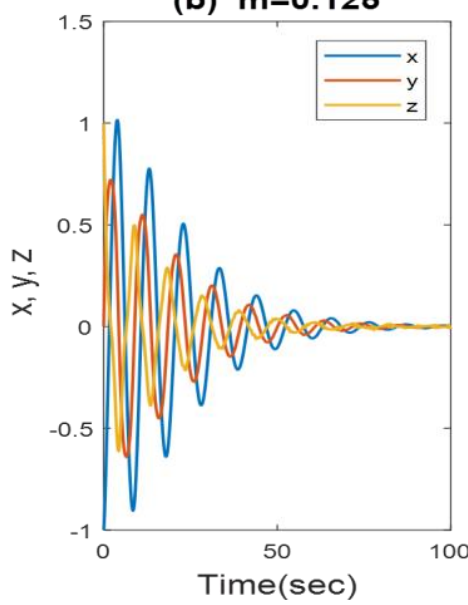

Figure 3. (a) Paths system before adding the control units (b) paths system after adding the control units

\subsection{Dislocated feedback control}

in this strategy (dislocated feedback control), we have six formulas, two of them achieved to suppress dynamical system, these strategies can be summarized in the following Table 1, Figure 4 shows successful strategies with positive feedback coefficient ( $\mathrm{Y}=-\mathrm{my}$ ) for system (5).

Table 1. Control their auxiliary equation and corresponding of index of Routh-Hurwitz criterion

\begin{tabular}{|c|c|c|c|c|}
\hline No & $\begin{array}{l}\text { Control } \\
\mathrm{Y}=\left[\mathrm{v}_{\mathrm{i}}\right]^{\mathrm{T}}\end{array}$ & auxiliary equation & Routh-Hurwitz criterion & $\begin{array}{l}\text { the conditions } \\
\text { Based of theorem }\end{array}$ \\
\hline 1. & {$[0,-\mathrm{my}, 0]^{\mathrm{T}}$} & $\tau^{3}+\alpha \tau^{2}-(\delta-m \beta) \tau+\beta=0$ & $\begin{array}{c}\mathrm{A}>0, \mathrm{C}>0 \\
A B-C>0 \Rightarrow m>0.3547\end{array}$ & the first condition \\
\hline 2. & {$[0,0,-\mathrm{my}]^{\mathrm{T}}$} & $\tau^{3}+\alpha \tau^{2}-(\delta-m \beta) \tau+\beta=0$ & $\begin{array}{c}\mathrm{A}>0, \mathrm{C}>0 \\
A B-C>0 \Rightarrow m>0.4611\end{array}$ & the first condition \\
\hline 3. & {$[-\mathrm{my}, 0,0]^{\mathrm{T}}$} & $\tau^{3}+\alpha \tau^{2}-\delta \tau-m \beta+\beta=0$ & $m_{1}<1, m_{2}>1.276$ & the four condition \\
\hline 4. & {$[0,-m x, 0]^{\mathrm{T}}$} & $\tau^{3}+\alpha \tau^{2}-\delta \tau-m \beta+\beta=0$ & $m_{1}<36, m_{2}>36.9765$ & the four condition \\
\hline 5. & {$[0,-\mathrm{mz}, 0]^{\mathrm{T}}$} & $\tau^{3}+\alpha \tau^{2}-\delta(1-m) \tau+\beta(1-m)=0$ & $m_{1}<1, m_{2}>1$ & the four condition \\
\hline
\end{tabular}

Corollary: When the control as $\left(\mathrm{Y}_{2}=-\mathrm{mx}\right)$ we have negative feedback coefficient failure this strategy.

(a) $\mathrm{m}=0.33$

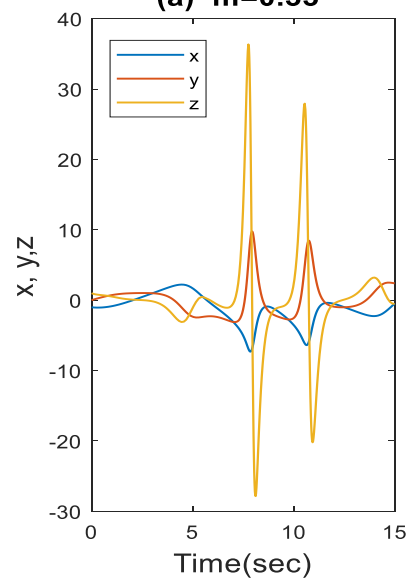

(b) $\mathrm{m}=0.36$

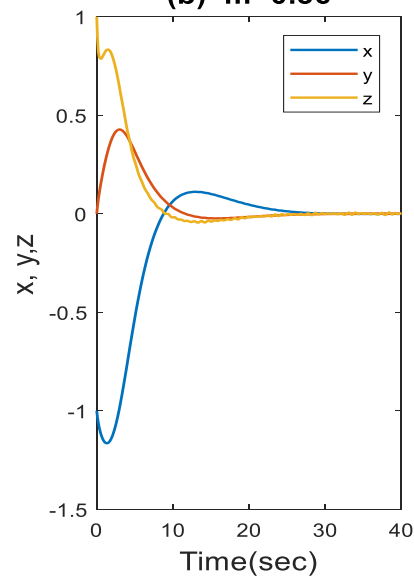

Figure 4. (a) Paths system before adding the control units (b) paths system after adding the control units 


\subsection{Speed feedback control}

In this method we have 6 formulas, two of them have been effective in repress the dynamic system, these strategies can be summarized in the following Table 2, Figure 5 shows successful strategies with positive feedback coefficient (m) for system (5).

Table 2. Control their auxiliary equation and corresponding of index of Routh-Hurwitz criterion

\begin{tabular}{|c|c|c|c|c|}
\hline No & Control $\mathrm{Y}=\left[\mathrm{v}_{\mathrm{i}}\right]^{\mathrm{T}}$ & auxiliary equation & Routh-Hurwitz criterion & $\begin{array}{l}\text { the conditions Based } \\
\text { of theorem }\end{array}$ \\
\hline 1. & {$[0,-m \dot{x}, 0]^{T}$} & $\tau^{3}+(\alpha+m) \tau^{2}+(\alpha m-\delta) \tau+\beta=0$ & $\begin{array}{c}\mathrm{A}>0, \mathrm{C}>0 \\
A B-C>0 \Rightarrow m \\
>0.1247 \\
\mathrm{~A}>0, \mathrm{C}>0\end{array}$ & the first condition \\
\hline 2. & {$\left[0,0,-m x^{\cdot}\right]^{T}$} & $\tau^{3}+\alpha \tau^{2}+(m-\delta) \tau+\beta=0$ & $\begin{array}{l}A B-C>0 \Rightarrow m \\
>0.4611\end{array}$ & the first condition \\
\hline $\begin{array}{l}3 . \\
4 .\end{array}$ & $\begin{array}{l}{[-m \dot{z}, 0,0]^{T}} \\
{[-m \dot{y}, 0,0]^{T}}\end{array}$ & $\begin{array}{c}\tau^{3}+\alpha \tau^{2}-(\delta+m \beta) \tau+\beta=0 \\
\tau^{3}+(\alpha-\beta m) \tau^{2}-\delta \tau+\beta+\beta \delta m=0\end{array}$ & $\begin{array}{l}m>-0.3547 \\
m>-1.4188\end{array}$ & $\begin{array}{l}\text { the second condition } \\
\text { the second condition }\end{array}$ \\
\hline 5. & {$[0,-m \dot{z}, 0]^{T}$} & $\tau^{3}+(\alpha+\delta m) \tau^{2}+(-\beta m-\delta) \tau+\beta=0$ & $\begin{array}{c}m_{1}>-35.7194 \\
m_{2}>-0.3574486\end{array}$ & the second condition \\
\hline 6. & {$[0,0,-m \dot{y}]^{T}$} & $\tau^{3}+(\alpha+m) \tau^{2}-\delta \tau+\beta=0$ & $m>-16.6$ & the second condition \\
\hline
\end{tabular}

(a) $m=0.450$

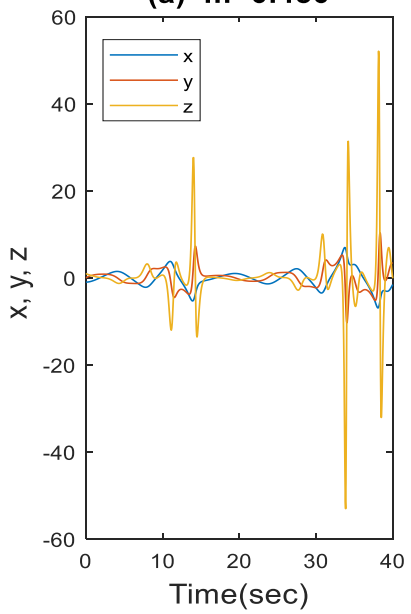

(b) $m=0.470$

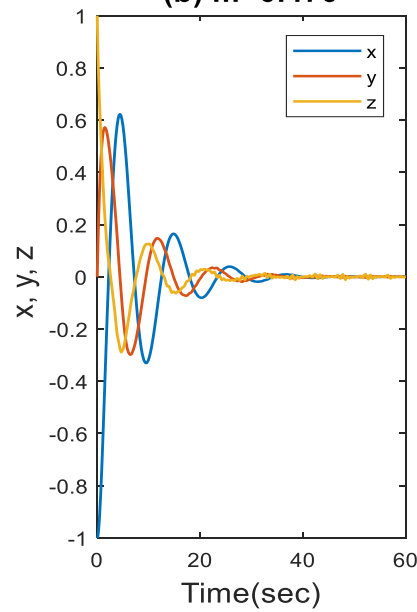

Figure 5. (a) Paths system before adding the control units (b) paths system after adding the control units

\subsection{Enhancing feedback control:}

By using this strategy (Enhancing feedback control), we obtained four terms, three of them subdued dynamic system, the following Table 3 briefly describes these strategies and the Figure 6 presentation of one of the felicitous strategies.

Table 3. Control their auxiliary equation and corresponding of index of Routh-Hurwitz criterion

\begin{tabular}{|c|c|c|c|c|}
\hline No & Control $\mathrm{Y}=\left[\mathrm{v}_{\mathrm{i}}\right]^{\mathrm{T}}$ & auxiliary equation & $\begin{array}{c}\text { Routh-Hurwitz } \\
\text { criterion }\end{array}$ & $\begin{array}{l}\text { the conditions Based } \\
\text { of theorem }\end{array}$ \\
\hline 1. & {$[-\mathrm{mx},-\mathrm{my}, 0]^{\mathrm{T}}$} & $\begin{array}{c}\tau^{3}+(\alpha+2 m) \tau^{2}+\left(2 \alpha m+k^{2}-\delta\right) \tau+\left(\alpha m^{2}\right. \\
-\delta m+\beta=0\end{array}$ & $\begin{array}{l}\mathrm{A}>0, \mathrm{C}>0 \\
A B-C>0 \Rightarrow m \\
>0.0621\end{array}$ & the first condition \\
\hline 2. & {$[-\mathrm{mx}, 0,-\mathrm{mz}]^{\mathrm{T}}$} & $\begin{array}{c}\tau^{3}+(\alpha+m) \tau^{2}+\left(\alpha m+m^{2}-\delta\right) \tau+(\beta \\
-\delta m)=0\end{array}$ & $\begin{array}{l}\mathrm{A}>0, \mathrm{C}>0 \\
A B-C>0 \Rightarrow m \\
>0.1172\end{array}$ & the first condition \\
\hline 3. & {$[0,-m y,-m z]^{\mathrm{T}}$} & $\begin{array}{c}\tau^{3}+(\alpha+m) \tau^{2}-\left(\alpha m+m^{2}+m-\delta\right) \tau+\beta \\
=0\end{array}$ & $\begin{array}{l}\mathrm{A}>0, \mathrm{C}>0 \\
A B-C>0 \Rightarrow m \\
>0.0961\end{array}$ & the first condition \\
\hline 4. & {$[-m x,-m y,-m z]^{T}$} & $\begin{aligned} \tau^{3}+(\alpha+3 m) \tau^{2}+ & \left(2 \alpha m+3 m^{2}-\delta\right) \tau \\
& +\left(\alpha m^{2}-\beta-\delta m=0\right.\end{aligned}$ & $\begin{array}{l}m_{1} \\
>-1.5809 \\
\pm 0.4874 i, m_{2} \\
>0.03815\end{array}$ & the second condition \\
\hline
\end{tabular}



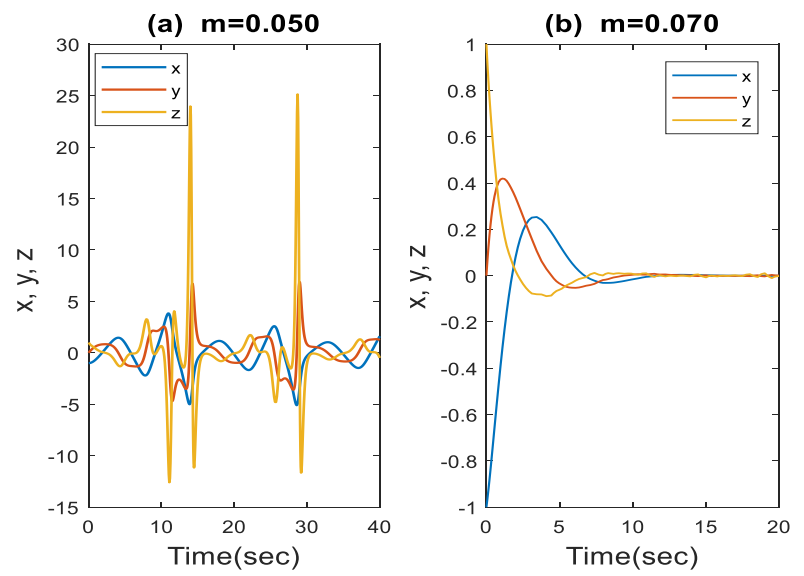

Figure 6. (a) Paths system before adding the control units (b) paths system after adding the control units

\subsection{Conbine between ordinary and dislocated feedback control}

In this technique, we combine between ordinary feedback control and dislocated feedback control, where six forms were get, but five of them were felicitous in obtaining on the coefficient of positive feedback for system (5), The following Table 4 summarizes the successful strategies we have obtained in repress the system, and Figure 7 shows one of the successful strategies

Table 4. Control their auxiliary equation and corresponding of index of Routh-Hurwitz criterion

\begin{tabular}{|c|c|c|c|c|}
\hline No & Control $\mathrm{Y}=\left[\mathrm{v}_{\mathrm{i}}\right]^{\mathrm{T}}$ & auxiliary equation & Routh-Hurwitz criterion & $\begin{array}{c}\text { the conditions Based } \\
\text { of theorem }\end{array}$ \\
\hline 1. & {$[(-\mathrm{mx},-\mathrm{my}), 0,0]^{\mathrm{T}}$} & $\begin{array}{r}\tau^{3}+(\alpha+m) \tau^{2}+(\alpha m-\delta) \tau+\beta \\
-\beta m-\delta m \\
=0\end{array}$ & $\begin{aligned} A>0 & \\
\mathrm{C}=1.3-0.1 \mathrm{~m}-1.3 \mathrm{~m} & >0 \\
& \Rightarrow m_{1} \\
& <0.9285\end{aligned}$ & the third condition \\
\hline 2. & {$[(-\mathrm{mx},-\mathrm{mz}), 0,0]^{\mathrm{T}}$} & $\begin{array}{c}\tau^{3}+(\alpha+m) \tau^{2}+(\alpha m-\delta-\beta m) \tau \\
-\delta m)=0\end{array}$ & $\begin{array}{c}A B-C>0 \Rightarrow m_{2}>0.1131 \\
A>0 \\
\mathrm{C}=1.3-0.1 * \mathrm{~m} \Rightarrow \mathrm{m}<13 \\
A B-C>0 \Rightarrow m>0.1904\end{array}$ & the third condition \\
\hline 3. & {$[0,(-\mathrm{mx},-\mathrm{my}), 0]^{\mathrm{T}}$} & $\begin{array}{r}\tau^{3}+(\alpha+m) \tau^{2}+(\alpha m+m-\delta) \tau \\
+\beta+\alpha m=0\end{array}$ & $\begin{array}{c}A>0, C>0 \\
A B-C>0 \Rightarrow m>0.1236\end{array}$ & the first condition \\
\hline 4. & {$[0,(-\mathrm{mx},-\mathrm{mz}), 0]^{\mathrm{T}}$} & $\begin{array}{r}\tau^{3}+(\alpha+m) \tau^{2}+(\alpha m-\delta+\delta m) \tau \\
+\beta-\beta m=0\end{array}$ & $\begin{array}{c}A>0 \\
C=1.3-1.3 m \Rightarrow m_{1}<1 \\
A B-C>0 \Rightarrow m_{2}>0.1111\end{array}$ & the third condition \\
\hline 5. & {$[0,0,(-m x,-m z)]^{\mathrm{T}}$} & $\tau^{3}+(\alpha+m) \tau^{2}+(m-\delta) \tau+\beta=0$ & $\begin{array}{c}A>0, C>0 \\
A B-C>0 \Rightarrow m>0.4231\end{array}$ & the first condition \\
\hline 6. & {$[0,0,(-\mathrm{mx},-\mathrm{mz})]^{\mathrm{T}}$} & $\tau^{3}+(\alpha+m) \tau^{2}-\delta \tau+\beta+m=0$ & $m>-1.5090$ & the second condition \\
\hline
\end{tabular}

(a) $\mathrm{m}=0.0911$

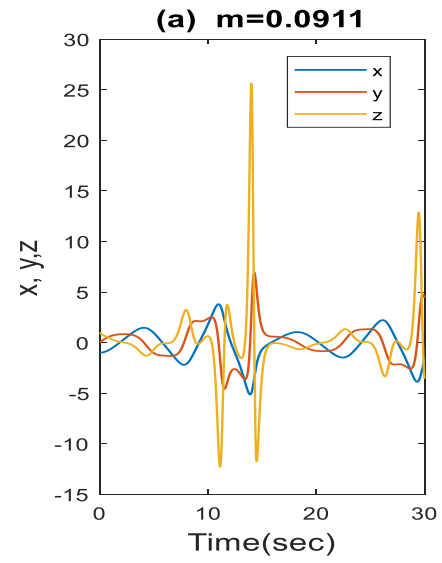

(b) $\mathrm{m}=0.1211$

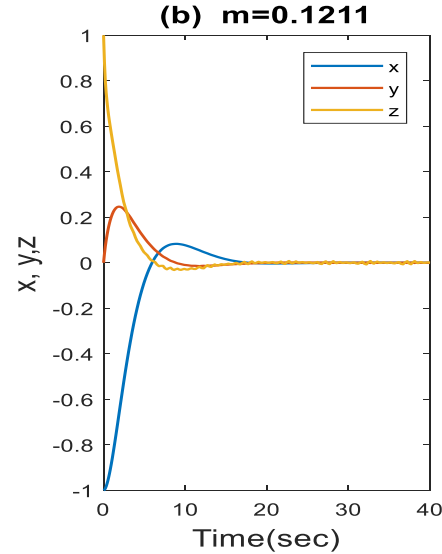

Figure 7. (a) paths system before adding the control units (b) paths system after adding the control units 


\subsection{Conbine between ordinary and speed feedback control}

In this technique, we combine between ordinary feedback control and dislocated feedback control, where six forms were get, but five of them were successful in obtaining the coefficient of positive feedback for system (5), The following Table 5 summarizes felicitous strategies obtained in suppressing the system, and Figure 8 shows one of the successful strategies.

Table 5. Control their auxiliary equation and corresponding of index of Routh-Hurwitz criterion

\begin{tabular}{|c|c|c|c|c|}
\hline No & Control $\mathrm{Y}=\left[\mathrm{v}_{\mathrm{i}}\right]^{\mathrm{T}}$ & auxiliary equation & Routh-Hurwitz criterion & $\begin{array}{l}\text { the conditions Based } \\
\text { of theorem }\end{array}$ \\
\hline 1. & {$[(-m x,-m \dot{y}), 0,0]^{\prime}$} & $\begin{array}{r}\tau^{3}+(\alpha+m) \tau^{2}+(\alpha m-\delta-\beta m) \tau \\
+\beta-\delta m=0\end{array}$ & $\begin{array}{c}A>0 \\
\mathrm{C}=1.3-0.1 \mathrm{~m}>0 \Rightarrow m_{1}<13 \\
A B-C>0 \Rightarrow m_{2}>0.1904\end{array}$ & the third condition \\
\hline 2. & {$[(-m x,-m \dot{z}), 0,0]^{7}$} & $\begin{aligned} \tau^{3}+(m-\beta m+\alpha) & \tau^{2}+(\alpha m-\delta) \tau \\
& +\beta-\delta m) \\
& =0\end{aligned}$ & $\begin{array}{c}A>0 \Rightarrow m_{1}<12 \\
\mathrm{C}=1.3-0.1 * \mathrm{~m} \Rightarrow m_{2}<13 \\
A B-C>0 \Rightarrow m_{2} \\
>0.1281(\text { and }) m_{3}<11.9922\end{array}$ & the third condition \\
\hline 3. & {$[0,(-m \dot{x},-m y), 0]^{T}$} & $\begin{array}{r}\tau^{3}+(2 m+\alpha) \tau^{2}+(2 \alpha m-\delta) \tau \\
+\beta=0\end{array}$ & $\begin{array}{c}A>0, C>0 \\
A B-C>0 \Rightarrow m>0.0623\end{array}$ & the first condition \\
\hline 4. & {$[0,(-m x,-m \dot{z}), 0]$} & $\begin{aligned} \tau^{3}+(m+\delta m+\alpha) \tau^{2} & \\
& +(\alpha m-\beta m \\
& -\delta) \tau+\beta=0\end{aligned}$ & $\begin{array}{c}A>0, C>0 \\
A B-C>0 \Rightarrow m>0.1917\end{array}$ & the first condition \\
\hline 5. & {$[0,0,(-m \dot{x},-m z)]^{1}$} & $\begin{array}{c}\tau^{3}+(\alpha+m) \tau^{2}+(m-1) \tau+\beta \\
=0\end{array}$ & $\begin{array}{c}A>0, C>0 \\
A B-C>0 \Rightarrow m>1.2670\end{array}$ & the first condition \\
\hline 6. & {$[0,0,(-m \dot{y},-m z)]^{T}$} & $\tau^{3}+(\alpha+2 m) \tau^{2}-\delta \tau+\beta=0$ & $m>-8.3$ & the second condition \\
\hline
\end{tabular}

(a) $m=0.1816$

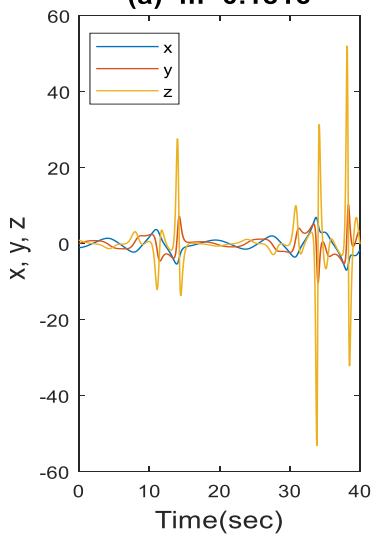

(b) $m=0.2$

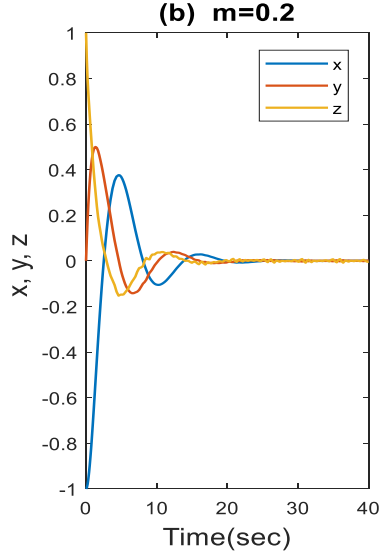

Figure 8. (a) paths system before adding the control units (b) paths system after adding the control units

In this section will be compared between (ordinary and dislocated feedback control) with (combine between strategies ordinary and dislocated feedback control) in the Table part (I) from the side and (ordinary and speed feedback control) with (combine between strategies ordinary and speed feedback control) in the Table part (II) from the other side, As shown in the next tables.

\begin{tabular}{|c|c|c|c|c|c|c|}
\hline \multicolumn{7}{|c|}{ Part (I) } \\
\hline \multicolumn{3}{|c|}{ Ordinary feedback control } & \multicolumn{2}{|c|}{ Dislocated feedback control } & \multicolumn{2}{|c|}{$\begin{array}{l}\text { Ccombine between strategies ordinary } \\
\text { and dislocated feedback control }\end{array}$} \\
\hline no & $\begin{array}{l}\text { Control } \\
\mathrm{Y}=\left[\mathrm{v}_{\mathrm{i}}\right]^{\mathrm{T}}\end{array}$ & $\begin{array}{l}\text { feedback } \\
\text { control }\end{array}$ & Control $\mathrm{Y}=\left[\mathrm{v}_{\mathrm{i}}\right]^{\mathrm{T}}$ & $\begin{array}{l}\text { feedback } \\
\text { control }\end{array}$ & Control $\mathrm{Y}=\left[\mathrm{v}_{\mathrm{i}}\right]^{\mathrm{T}}$ & $\begin{array}{l}\text { feedback } \\
\text { control }\end{array}$ \\
\hline 1. & $\begin{array}{l}{\left[0,-\mathrm{mx}_{2}\right.} \\
, 0]^{\mathrm{T}}\end{array}$ & $m>0.1247$ & {$\left[0,-\mathrm{mx}_{2}, 0\right]^{\mathrm{T}}$} & $m>0.35474$ & {$\left[\left(-\mathrm{mx}_{1},-\mathrm{mx}_{2}\right), 0,0\right]^{\mathrm{T}}$} & $\begin{array}{l}m_{1}<0.9285 \\
m_{2}>0.1131\end{array}$ \\
\hline 2. & & & {$\left[0,0,-\mathrm{mx}_{2}\right]^{\mathrm{T}}$} & $m>0.4611$ & {$\left[\left(-\mathrm{mx}_{1},-\mathrm{mx}_{3}\right), 0,0\right]^{\mathrm{T}}$} & $\begin{array}{c}\mathrm{m}_{1}<13 \\
m_{2}>0.1904\end{array}$ \\
\hline 3. & $\ldots \ldots \ldots$ & …..... & ........ & $\ldots \ldots \ldots$ & {$\left[0,\left(-\mathrm{mx}_{1},-\mathrm{mx}_{2}\right), 0\right]^{\mathrm{T}}$} & $m>0.1236$ \\
\hline 4. & $\ldots \ldots \ldots$ & $\ldots \ldots \ldots$ & $\cdots \cdots \cdots$ & - n...... & {$\left[0,\left(-\mathrm{mx}_{1},-\mathrm{mx}_{3}\right), 0\right]^{\mathrm{T}}$} & $\begin{array}{c}m_{1}<1 \\
m_{2}>0.1111\end{array}$ \\
\hline 5. & $\ldots \ldots$. & ........ & & ........ & {$\left[0,0,\left(-\mathrm{mx}_{1},-\mathrm{mx}_{3}\right)\right]^{\mathrm{T}}$} & $m>0.4231$ \\
\hline
\end{tabular}




\begin{tabular}{|c|c|c|c|c|c|c|}
\hline \multicolumn{7}{|c|}{ Part (II) } \\
\hline & \multicolumn{2}{|c|}{ Ordinary feedback control } & \multicolumn{2}{|c|}{ Speed feedback control } & \multicolumn{2}{|c|}{$\begin{array}{c}\text { Combine between strategies ordinary and } \\
\text { speed feedback control }\end{array}$} \\
\hline $\begin{array}{l}\mathrm{N} \\
\mathrm{o}\end{array}$ & $\begin{array}{l}\text { Control } \\
\mathrm{V}=\left[\mathrm{v}_{\mathrm{i}}\right]^{\mathrm{T}}\end{array}$ & $\begin{array}{c}\text { feedback } \\
\text { control }\end{array}$ & $\begin{array}{l}\text { Control } \\
\mathrm{V}=\left[\mathrm{v}_{\mathrm{i}}\right]^{\mathrm{T}}\end{array}$ & $\begin{array}{c}\text { feedback } \\
\text { control }\end{array}$ & Control $\mathrm{V}=\left[\mathrm{v}_{\mathrm{i}}\right]^{\mathrm{T}}$ & $\begin{array}{c}\text { feedback } \\
\text { control }\end{array}$ \\
\hline 1. & {$\left[0,-\mathrm{mx}_{2}, 0\right]^{\mathrm{T}}$} & $m>0.1247$ & {$\left[0,-m \dot{x_{1}}, 0\right]^{\mathrm{T}}$} & $m>0.1247$ & {$\left[\left(-m x_{1},-m \dot{x}_{2}\right), 0,0\right]^{T}$} & $\begin{array}{c}m_{1}<13 \\
m_{2}>0.1904 \\
m_{1}<12\end{array}$ \\
\hline 2. & an.... & -....... & {$\left[0,0,-m \dot{x_{1}}\right]^{\mathrm{T}}$} & $m>0.4611$ & {$\left[\left(-m x_{1},-m \dot{x}_{3}\right), 0,0\right]^{T}$} & $\begin{array}{l}m_{2}<13 \\
m_{3} \\
<11.9922\end{array}$ \\
\hline 3. & $\ldots \ldots \ldots$ & $\ldots \ldots \ldots$ & ........ & ….... & {$\left[0,\left(-m \dot{x}_{1},-m x_{2}\right), 0\right]^{T}$} & $m>0.0623$ \\
\hline 4. & ......... & ......... & ......... & ......... & {$\left[0,\left(-m x_{2},-m \dot{x}_{3}\right), 0\right]^{T}$} & $m>0.1917$ \\
\hline 5. & $\ldots \ldots \ldots$ & $\ldots \ldots \ldots$ & $\ldots \ldots \ldots$ & ........ & {$\left[0,0,\left(-m \dot{x}_{1},-m x_{3}\right)\right]^{T}$} & $m>1.2670$ \\
\hline
\end{tabular}

\section{CONCLUSIONS}

In this paper, we referred to the linear feedback control strategies, which included (ordinary, dislocated, enhancing feedback control) addition to the speed feedback control strategy on the Jerk system. we found that the enhancing strategy got the best results because it contains more than one feedback. For this reason, we combined these previous strategies to improve our results. When compared with the original strategies, it was found that the method of integration is efficient and distinct and can be applied on other dynamic systems.

\section{ACKNOWLEDGEMENTS}

The authors is very grateful to the University of Mosul /College of Computer Sciences and Mathematics, for their provided facilities, which helped to improve the quality of this work.

\section{REFERENCES}

[1] C. Tao, et al., "Speed Feedback Control of Chaotic System," Chaos Solitons Fractals, vol. 23, pp. 259-263, 2005.

[2] S. F. Al-Azzawi, et al., "Chaotic Lorenz System and it's Suppressed," Journal of Advanced Research in Dynamical and Control Systems, vol.12, no. 2, pp. 548-555, 2020.

[3] A. S. Al-Obeidi and S. F. Al-Azzawi, "Chaos Synchronization in a 6-D Hyperchaotic System with Self-Excited Attractor," TELKOMNIKA (Telecommunication, Computing, Electronics and Control), vol. 18, no 3, pp. 1483-1490, June 2020.

[4] Z. N. Al-Khatee and S J. Mohammedand, "A Novel Approach for Audio File Encryption Using Hand Geometry," Multimedia Tools and Applications, Vol. 79, No. 11-12,Mar 2020.

[5] S. F. Al-Azzawi, "Stability and Bifurcation of Pan Chaotic System by Using Routh-Hurwitz and Gardan Method," Applied Mathematics and Computation, vol. 219, no. 3, pp. 1144-1152, Oct 2012.

[6] Maysoon M. Aziz and Saad Fawzi AL-Azzawi, "Linear and Non-linear Feedback Control Strategies for a 4D Hyperchaotic System,” Pure and Applied Mathematics Journal, vol. 6, no. 1, pp. 5-13, 2017.

[7] C. Tao and C. Yang, "Three Control Strategies for the Lorenz Chaotic System," Chaos Solitons Fractals, vol. 35, pp. 1009-1014, 2008.

[8] C. Zhu, "Feedback Control Method for Stabilizing Unstable Equilibrium Points in a New Chaotic System," Nonlinear analysis, vol. 71, pp. 2441-2446, 2009.

[9] C. Zhu, Z. Chen, Feedback Control Strategies for the Liu Chaotic System, Phys. Lett. A, vol.372, pp. 4033-4036, 2008.

[10] C. Zhu, "Controlling Hyper Chaos in Hyperchaotic Lorenz System Using Feedback Controllers, Applied Mathematics and Computation, vol. 216, pp. 3126-3132, 2010.

[11] Maysoon M. Aziz, Saad Fawzi AL-Azzawi, "Using Feedback Control Methods to Suppress a Modified Hyperchaotic Pan System", Computational and Applied Mathematics Journal, vol.1, no 3, pp. 97-106, 2015.

[12] Z. Sh. Al-Talib and S. F. AL-Azzawi, "Projective Synchronization for 4D Hyperchaotic System Based on Adaptive Nonlinear Control Strategy," Indonesian Journal of Electrical Engineering and Computer Science, vol. 19, no. 2, Aug 2020 .

[13] A. Sambas, et al., "Design and Numerical Simulation of Unidirectional Chaotic Synchronization and its Application in Secure Communication System", Journal of Engineering Science and Technology Review, vol. 6, no. 4, pp. 66-73, 2013.

[14] M. Zhang and Q. Han, "Dynamic analysis of an Autonomous Chaotic System with Cubic Nonlinearity, Optik, vol. 127 , no. 10, pp. 4315-4319, 2016.

[15] A. S. Al-Obeidi and S. F. Al-Azzawi, "Complete Synchronization of a Novel 6-D Hyperchaotic Lorenz System with Known Parameters," International Journal of Engineering \& Technology, vol. 7, no.4, pp. 5345-5349, 2018.

[16] S. F. Al-Azzawi and M. M. Aziz, "Strategies of Linear Feedback Control and Its Classification," TELKOMNIKA (Telecommunication, Computing, Electronics and Control), vol. 17, no. 4, pp. 1931-1940, Aug 2019. 
[17] M. A. A. Alhafedh and O. S. Qasim, "Two-Stage Gene Selection in Microarray Dataset Using Fuzzy Mutual Information and Binary Particle Swarm Optimization," Indian Journal of Forensic Medicine \& Toxicology, vol. 13, pp. 1162-1171, 2019.

[18] Z. Sh. Al-Talib and S. F. AL-Azzawi, "Projective and Hybrid Projective Synchronization of 4-D Hyperchaotic System via Nonlinear Controller Strategy," TELKOMNIKA (Telecommunication, Computing, Electronics and Control), vol. 18, no 2, pp. 1012-1020, April 2020.

[19] S. Y. Al-hayali and S. F. AL-Azzawi, "An Optimal Nonlinear Control for Anti-Synchronization of Rabinovich Hyperchaotic System," Indonesian Journal of Electrical Engineering and Computer Science (IJEECS), vol. 19, no. 1, pp. 379-386, July 2020.

[20] A. S. Al-Obeidi and S. F. Al-Azzawi, "Projective Synchronization for a Class of 6-D Hyperchaotic Lorenz System," Indonesian Journal of Electrical Engineering and Computer Science (IJEECS), vol. 16, no. 2, pp. 692-700, Nov 2019.

[21] S. Y. Al-hayali and S. F. AL-Azzawi, "An Optimal Control for Complete Synchronization of 4D Rabinovich Hyperchaotic Systems," TELKOMNIKA (Telecommunication, Computing, Electronics and Control), vol. 18, no 2, pp. 994-1000, Apr 2020.

[22] K. A. Abed and A. A. Ahmad, "The Best Parameters Selection Using Pso Algorithm to Solving For Ito System by New Iterative Technique," Indonesian Journal of Electrical Engineering and Computer Science (IJEECS), vol. 18, no. 3, pp. 1638-1645, June 2020.

[23] A. F. Qasim and B. J. Salim, "Application New Iterative Method for Solving Modified Korteweg-Devries (MKdV) System from Three Equations," Journal of Advanced Research in Dynamical and Control Systems, vol.11, pp.1-7, 2019.

[24] Z. N. Al-Khateeb and M. F. Jader, "Encryption and Hiding Text Using DNA Coding and Hyperchaotic System," Indonesian Journal of Electrical Engineering and Computer Science (IJEECS), vol. 19, no. 2, Aug 2020.

[25] S. F. Al-Azzawi and M. M. Aziz, "Chaos Synchronization of Nonlinear Dynamical Systems via a Novel Analytical Approach,” Alexandria Engineering Journal, vol. 57, no. 4, pp. 3493-3500, Dec 2018.

[26] M. M. Aziz and S. F. Al-Azzawi, "Anti-synchronization of Nonlinear Dynamical Systems Based on Gardano's Method," Optik, vol. 134, pp. 109-120, Apr 2017.

[27] M. M. Aziz and S. F. Al-Azzawi, "Hybrid Chaos Synchronization between Two Different Hyperchaotic Systems via Two Approaches,” Optik, vol. 138, pp. 328-340, Jun 2017. 